\title{
GALEX observations of quasar variability in the ultraviolet
}

\author{
B. Y. Welsh ${ }^{1,3}$, J. M. Wheatley ${ }^{1,3}$, and J. D. Neil ${ }^{2}$ \\ 1 Space Sciences Laboratory, University of California, 7 Gauss Way, Berkeley, CA 94720, USA \\ e-mail: bwelsh@ssl. berkeley.edu \\ 2 California Institute of Technology, MC 405-47, 1200 East California Boulevard, Pasadena, CA 91125, USA \\ 3 Eureka Scientific Inc., 2452 Delmer Street, Oakland, CA 94602, USA
}

Received 4 October 2010 / Accepted 14 December 2010

\begin{abstract}
Aims. Using archival observations recorded over a 5+ year timeframe with the NASA GALaxy Evolution eXplorer (GALEX) satellite, we present a study of the ultraviolet (UV) variability of 4360 quasars of redshifts up to $z=2.5$ that have optical counterparts in the Sloan Digital Sky Survey DR5 spectroscopic catalog of Schneider et al. (2007, AJ, 134, 102). The observed changes in both the far UV (FUV: 1350-1785 ̊) and near UV (NUV: 1770-2830 ̊̊) $A B$ magnitudes as a function of time may help differentiate between models of the emission mechanisms thought to operate in these active galaxies.

Methods. A list of NUV and FUV variable quasars was derived from the UV light-curves of sources with 5 or more observational visits by GALEX that spanned a time-frame $>3$ months. By measuring the error in the derived mean UV magnitude from the series of GALEX observations for each source, quasars whose UV variability was greater than the 3- $\sigma$ variance from the mean observed value were deemed to be (intrinsically) UV variable. This conservative selection criterion (which was applied to both FUV and NUV observations) resulted in identifying $550 \mathrm{NUV}$ and $371 \mathrm{FUV}$ quasars as being statistically significant UV variable objects.

Results. Following the work of Vanden Berk et al. (2004, ApJ, 601, 692), we have performed a structure function (SF) analysis of these data to search for possible correlations between UV variability and parameters such as rest frame time-lag, redshift, luminosity and radio loudness. Firstly, we observe that the amplitudes of variability as a function of time-lag for both the NUV and FUV data are far larger than those observed at visible wavelengths. Secondly, the levels of FUV variability are greater than those observed in the NUV for a given value of time-lag. Also, the amplitudes of both the NUV and FUV variability of quasars increase as a function of rest frame time-lag, irrespective of the value of quasar redshift, for time-lags $<200$ days. For time-lags $>300$ days there is a pronounced rollover in the NUV SF for all redshift values, which is also observed (with a lower signiPcance) in the FUV variability data. Although we find no strong relationship between UV variability and redshift, our data do show that higher redshift quasars appear to be more variable than their low redshift counterparts. Our data also show that, for all values of time-lag, the more luminous quasars tend to be slightly less UV variable, with perhaps the exception of FUV variable quasars for short time-lags.

Although our data sample is small, we find that radio-loud quasars are marginally more variable than radio quiet ones by a factor $\sim 2$ in the NUV and by a factor 1-3 in the FUV. Therefore, our present observations support the notion in which the radio properties of quasars have a limited influence on the observed UV variability of these objects. In summation, our present analysis favors a quasar model in which UV variability is mainly due to stochastic changes in the underlying continuum level, rather than models that favor gravitational microlensing or discrete-event processes.
\end{abstract}

Key words. ultraviolet: galaxies - quasars: general

\section{Introduction}

The flux emitted from quasars and other active galactic nuclei (AGN) such as Seyferts, BL Lacs and Blazars has been found to vary aperiodically on time-scales from hours to decades and from X-ray to radio wavelengths. In the case of radio-loud and radio-quiet quasars optical flux changes over timescales as short as minutes and with amplitudes ranging from a few hundredths to a few tenths of a magnitude (often refereed to as "optical microvariability"), have been widely reported (Miller et al. 1989; Stalin et al. 2005). The mechanism(s) responsible for this shortterm microvariability is still debated, with much of the visible spectral variability being explained by thermal processes associated with a black-hole accretion disk and/or non-thermal processes associated with the relativistic beaming of a jet (Ramirez et al. 2010; Pereyra et al. 2006). In fact Sesar et al. (2007) have found that nearly all quasars vary at some level when observed over long enough times scales, with $\sim 90 \%$ of quasars found in the Sloan Digital Sky Survey (SDSS) varying by $>0.03$ mag over a six year time scale at visible wavelengths. Although the actual cause of (long-term) variability in these objects is also still a matter of intense debate, variability studies of these sources are important since (in principal) they can set limits on the size of the emitting regions and can also constrain models of the emission mechanisms thought to operate in these active galaxies.

Numerous variability studies have been aimed at establishing correlations between quasar visible variability and important physical parameters such as source luminosity, redshift, time-lag (i.e. the time period between two sets of observations) and radio loudness (Helfand et al. 2001; Vanden Berk et al. 2004; de Vries et al. 2005; Ramirez et al. 2009). The establishment of such correlations is important since there is increasing evidence that the source of quasar variability is linked to the accretion disk surrounding the central supermassive black hole engine (Peterson et al. 2004; Pereyra et al. 2006; Wold et al. 2007). For example, Bachev (2009) has studied the observed time lags between the continuum emission from several optical bands for a large sample of quasars. He demonstrates that the lags are broadly 
consistent with an emission model in which the optical variations are largely due to the reprocessing of the central X-ray radiation in a surrounding thin accretion disk. However, both Kelly et al. (2009) and MacLeod et al. (2010), using a "damped random walk model", have suggested that these optical emission variations result from thermal fluctuations in the accretion disk that are powered by an underlying stochastic process such as a turbulent magnetic field. Clearly there is still much debate as to the interpretation of these optical variability studies (Kozlowski et al. 2010), and other emission mechanisms such as bursts of supernovae events close to the nucleus and extrinsic variations due to line-of-sight microlensing events cannot (as yet) be totally discounted (Ulrich et al. 1997). It is mostly agreed that the level of quasar variability increases with decreasing rest wavelength, which is related to the observation that their spectra become bluer in their bright phases. Several authors have claimed a variability-redshift correlation in the visible, although the actual sign of the correlation in the studies is debated (Giveon et al. 1999; Trevese \& Vagnetti 2002). For example, Vanden Berk et al. (2004) have claimed evidence for redshift evolution in quasar variability, with greater variability occurring at higher redshifts. However, such a trend is only revealed when an allowance is made for rest wavelength, absolute magnitude and time lag for each redshift interval. Such corrections to the observed optical variability data are crucial, since spectral features may move in and out of the photometric pass-bands with changes in the target redshift.

Quasars and AGN have also been well studied at ultraviolet (UV) wavelengths, such that typically their far UV spectra appear as several strong emission lines (formed in gas located further away from a central black hole and excited by continuum radiation) superposed on an underlying accretion-driven continuum. In contrast, their near UV spectra contain only a UV continuum with a "bump" at $2800 \AA$ superposed with the MgII emission lines (Vanden Berk et al. 2001). The UV variability of these objects has been widely reported, particularly with reference to reverberation mapping observations that in principal allow measurement of the size of the broad emission-line region and can infer the mass of the alleged central black hole (Peterson et al. 1993; Kaspi et al. 2000, 2007). UV photons are generally believed to be radiated from the quasar supermassive black hole accretion disk when its gravitational binding energy becomes released, while X-rays are produced in the disk corona. A statistical survey of the UV variability of 93 quasar/AGN, as observed with the IUE satellite, was carried out by Paltani \& Courvoisier (1994) who found no strong evidence for correlations between variability and parameters such as redshift, luminosity or UV spectra-index. However, changes and instabilities in the accretion disk must surely be linked to their observed UV variability, but recognizing and understanding the emission signatures from such features still remains problematic.

To proceed further with studies of UV variability we require access to far larger data sets than previously possible, and with the launch of the NASA GALaxy Evolution eXplorer (GALEX) satellite (Martin et al. 2005) we now have a significantly large database of far UV (FUV: 1350-1785 A) and near UV (NUV: 1770-2830 ̊) observations of quasars and AGNs that cover a timespan of over 5 years. Trammell et al. (2007) have presented a preliminary analysis of the basic UV properties of 6371 SDSS Data Release 3 (DR3) selected quasars observed during the first few years of the GALEX mission. One major conclusion from this study was that $>80 \%$ of the DR3 listed quasars had a corresponding GALEX NUV detection, implying that many more quasars should be detected during the remaining years of operation of the GALEX satellite. Bianchi et al. (2009) have shown that GALEX is also ideal for detecting quasars with blue (FUVNUV) colors that are difficult to identify from visible observations alone. Since the publication of these initial studies, newer and far larger versions of both the SDSS and GALEX catalogs are now in-hand. In particular, the catalog of quasars contained in the SDSS Data Release 5 (DR5) published by Schneider et al. (2007) lists some $77000+$ objects (with associated redshift and luminosity values), the majority of which should have UV counterparts observable with GALEX. Furthermore, since many quasars are known to be variable, this potentially enables us to study the UV variability of a significantly large number of these sources.

The largest group of known variable sources listed in the GALEX UV Variability-2 catalog are active galaxies (Wheatley et al. 2008). This sample of sources was derived solely through selection due to their observed UV variability, without prior knowledge that such sources were in fact AGN. These variable sources possessed redshifts as high as $z \sim 2.5$ and flux changes as large as $\sim 2.3 A B$ mag. were found in both of the GALEX near and far UV bands for AGN that were repeatedly observed over a 3.5 year time period. Based on the apparent quality of these data, we decided to search the entire GALEX archival database (which now spans 5+ years of GALEX observations) for spatial matches to quasars previously identified and listed in the SDSS DR5 catalog and then, for those sources that had multiple visit measurements, determine which ones exhibited measurable UV variability. This Paper discusses which GALEX sources were identified with SDSS quasar counterparts, which ones showed long-term UV variability (as measured over timeframes $>3$ months) and which, if any, correlations exist between this UV variability and other astrophysically interesting source parameters.

\section{Data analysis}

In order to produce a list of quasars that was suitable for an ultraviolet variability study we first searched the GALEX GR5 data archival release (at the Multi-Mission Archive at the Space Telescope Institute, MAST) for sources that spatially matched optically identified quasars listed in the SDSS DR5 spectroscopic catalog of Schneider et al. (2007). A spatial match was deemed positive if the central position of the matching GALEX source was within \pm 8 arcsec of the listed SDSS position, this number being slightly larger than the positional error of the GALEX observations for a point source detection. This search resulted in 77249 quasars that were common to both the SDSS and GALEX ultraviolet source catalogs.

In order to produce a list of quasars whose UV variability could be accurately assessed as a function of time, we then down-selected the large list of UV-selected quasars using the following criteria. Firstly, we only selected quasars with either (i) GALEX observations in the All-sky Imaging Survey (AIS) that were brighter than $\mathrm{NUV}_{\text {mag }}=19.0$ and with an exposure time of at least $100 \mathrm{~s}$; or (ii) those quasars with GALEX Deep Imaging Survey (DIS) observations of $1500 \mathrm{~s}$ duration or more (see Morrissey et al. 2007, for a detailed discussion of the observational modes of the GALEX satellite). A further restriction was applied such that only those quasars that had 5 or more observational visits by GALEX (and were detected in at least one of those visits) were selected. We note that an observational visit by GALEX does not necessarily result in a detection, since an intrinsically variable object may fall below the detection limit 


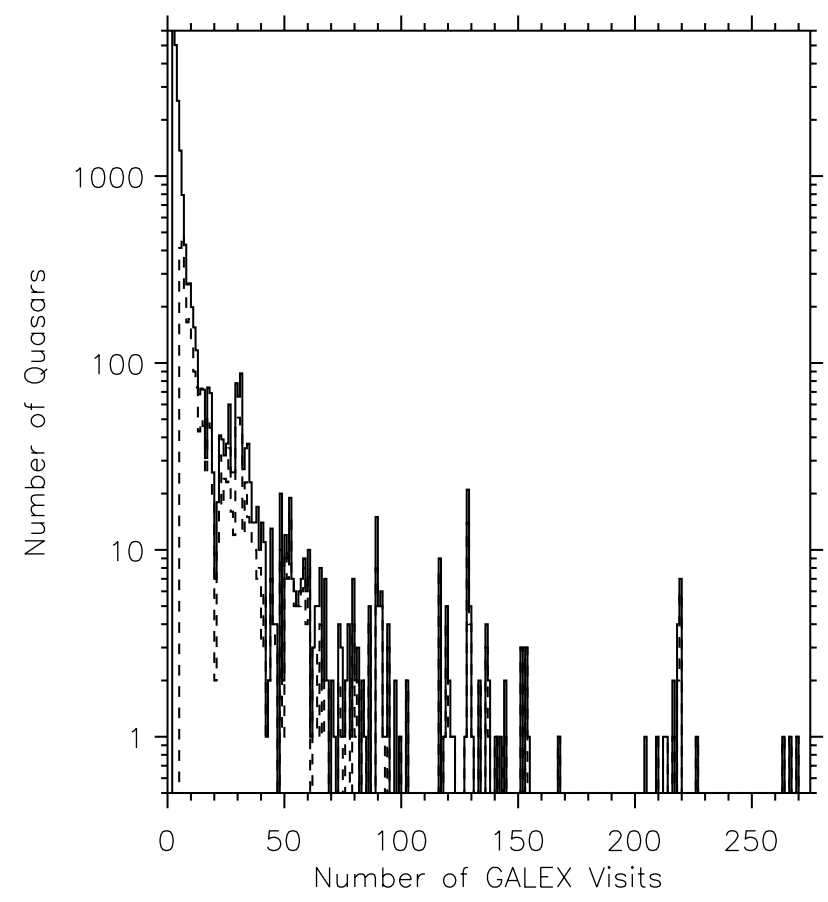

Fig. 1. Plot of the number of GALEX repeated visits for quasars with DIS integration times of $>1000 \mathrm{~s}$ and bright quasars with AIS observation of $>100 \mathrm{~s}$ (see text for selection criteria). Full line is for quasars with GALEX NUV observations and dashed line is for quasars with FUV observations.

throughout a particular visit. We were guided in these choices by Fig. 4 of Trammel et al. (2007) which shows the typical rms magnitude errors for an AIS and DIS observation with GALEX. Those results demonstrate that the measurement error for a typical AIS observation of a relatively UV bright $\left(\mathrm{NUV}_{\text {mag }}=19.0\right)$ object is approximately equal to the measurement error for a $1550 \mathrm{~s}$ DIS observation of a faint $\left(\mathrm{NUV}_{\mathrm{mag}}>21.0\right)$ source. In Fig. 1 we show a plot of the number of these selected quasars as a function of the number of observations in the GALEX NUV and FUV channels. It can be seen that there is a significant number of sources that have been visited between 5 and 20 times, with several sources being repeatedly observed $>100$ times. On application of the selection criteria of both exposure time and $\geq 5$ visits, this resulted in a list of 4360 quasars that were detected in at least one observational visit by GALEX.

In Fig. 2 we show a plot of these selected quasars as a function of the timespan covering all of their $4360 \mathrm{NUV}$ and 2709 FUV GALEX observational visits (NB: we note that the sample of FUV selected quasars is smaller than the number of NUV selected ones due to operational constraints on the FUV detector that often precluded simultaneous FUV and NUV observations of a source). This figure demonstrates that our quasar sample is not biased towards any particular timeframe of variability, with the majority of selected sources having been repeatedly sampled over a 1 to 5 year timespan. We note that although the time between many observational visits to a particular quasar may span several years, the target may be repeatedly observed on time-scales $<1$ day during a series of observational visits with GALEX.

All the data shown in Figs. 1 and 2 clearly indicate that our sample of quasars is both large and contains significant numbers of objects that have multiple $(\geq 5)$ observational visits spread over a significantly long timespan. In order to ensure that quasars with potentially short-term variations of a few days

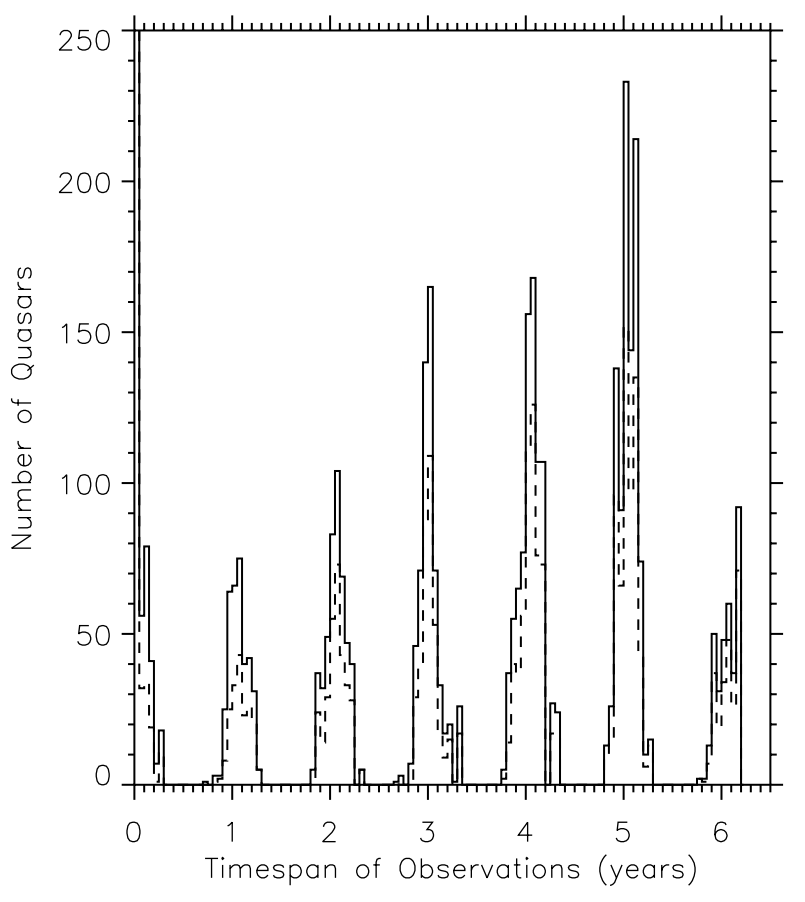

Fig. 2. Plot of the number UV selected quasars and the time period spanning all of their GALEX observations. Full line is for quasars observed in the GALEX NUV pass-band, dashed line is for quasars observed in the FUV pass-band.

had $\geq 5$ observations, we have further restricted our target selection to those ones whose visits spanned a time-frame $>3$ months. This selection criterion resulted in a final list of 3521 potentially UV variable quasars, of which 1942 had 10 or more observational visits. To ensure that all of the UV-selected sources were not in any preferential direction on the sky, in Fig. 3 we show the galactic locations of our SDSS selected sample that have been observed 5 or more times. Since our selection of targets was derived from the (northern hemisphere) SDSS survey cata$\log$, clearly only quasars with declinations $>-12^{\circ}$ are available for present study. Although there are large areas of the sky where we have no UV-selected quasars, Fig. 3 shows no obvious sightline preference apart from most sources being observed at high galactic latitude away from the effects of absorption of ultraviolet flux by gas and dust in the galactic plane. We then searched this list of 3521 sources for the ones which possessed statistically significant UV variability between each of their observational visits. Although the quasar nature of these sources at visible wavelengths was already known from the SDSS DR5 catalog, we decided that statistical confirmation of their associated UV variability was also required for this study. Due to the limited sensitivity of the GALEX observations this naturally restricted our study to the more UV variable quasars in this sample.

\subsection{UV variability selection}

In order to determine which of the 3521 selected quasars were variable at least once during their series of observations, we require knowledge of the size of the error associated with the estimation of each of the NUV and FUV magnitudes measured for each visit to a particular source. This error in this measurement is affected by the length of the observation by GALEX, the position of the source on the UV detector and the associated varying sky background signal which are both dependent on the (changing) 


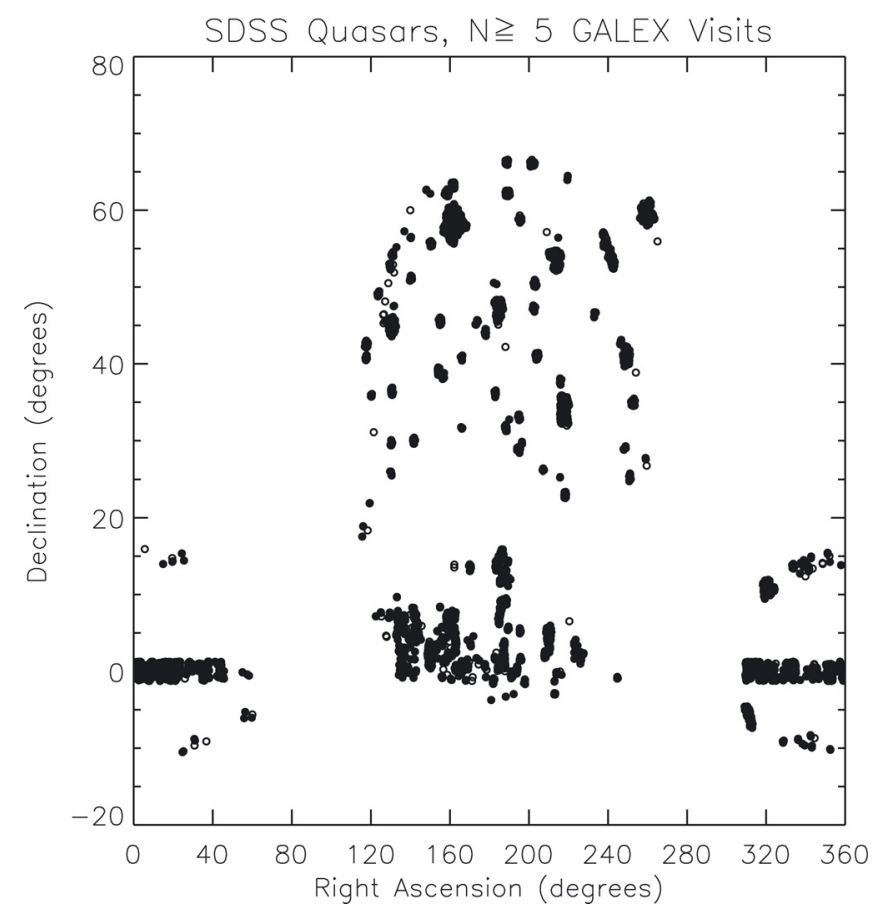

Fig. 3. Galactic distribution of the UV selected quasars with $\geq 5$ observational visits by GALEX. Filled circles are quasars with both NUV and FUV observations, while open circles are quasars with NUV only observations by GALEX.

orbital viewing characteristics of the GALEX satellite. Thus, in order to progress further in our study we clearly require a more consistent estimation of the measurement error for the UV magnitudes derived from each observational visit by the GALEX satellite. Fortunately, Gezari et al. (2008) have established a robust numerical method of assessing the statistical significance of the variability of a series of GALEX UV observations of the nuclei of normal quiescent galaxies, which is directly applicable to our present QSO data set. The method involves deriving the quantity, $\sigma(\langle m\rangle)$, which is defined as the standard deviation $(\sigma)$ from the weighted mean $(\langle m\rangle)$ of a series of observed UV magnitudes $(m)$. For a series of observations of the same object, the quantity of $\sigma(\langle m\rangle)$ will vary such that above some statistical level any observed change will be due to intrinsic source variability. Gezari et al. (2008) estimated the Poisson errors for a series of UV magnitude determinations using GALEX (see Eq. (2) in that paper). They found that the measured standard deviation from a mean UV source magnitude was dominated by systematic errors that were not accounted purely by the photometric error. We note that the major contribution to the error in the estimation of a UV magnitude using GALEX is thought to be due to the (often varying) sky background level. Thus to be conservative, Gezari et al. (2008) used a cut of 5- $\sigma$ above the mean magnitude level to determine intrinsic source variability.

Values of $\sigma(\langle m\rangle)$ for our quasar sample were also derived from Eq. (2) of Gezari et al. (2008) for each set of observational visits. The values of NUV and FUV $A B$ magnitudes recorded for each visit to a selected quasar, together with their measurement errors, were retrieved from the GALEX GR5 data archival release at the MAST. We used circular aperture magnitudes of a 6 arcsec radius, as listed in the MAST catalog, which were obtained using the GALEX pipe-line generated SExtractor magnitude estimator (Bertin \& Arnouts 1996). In Fig. 4 we show a plot of $\sigma(\langle m\rangle)$ versus $\langle m\rangle$ for the multiple NUV observations

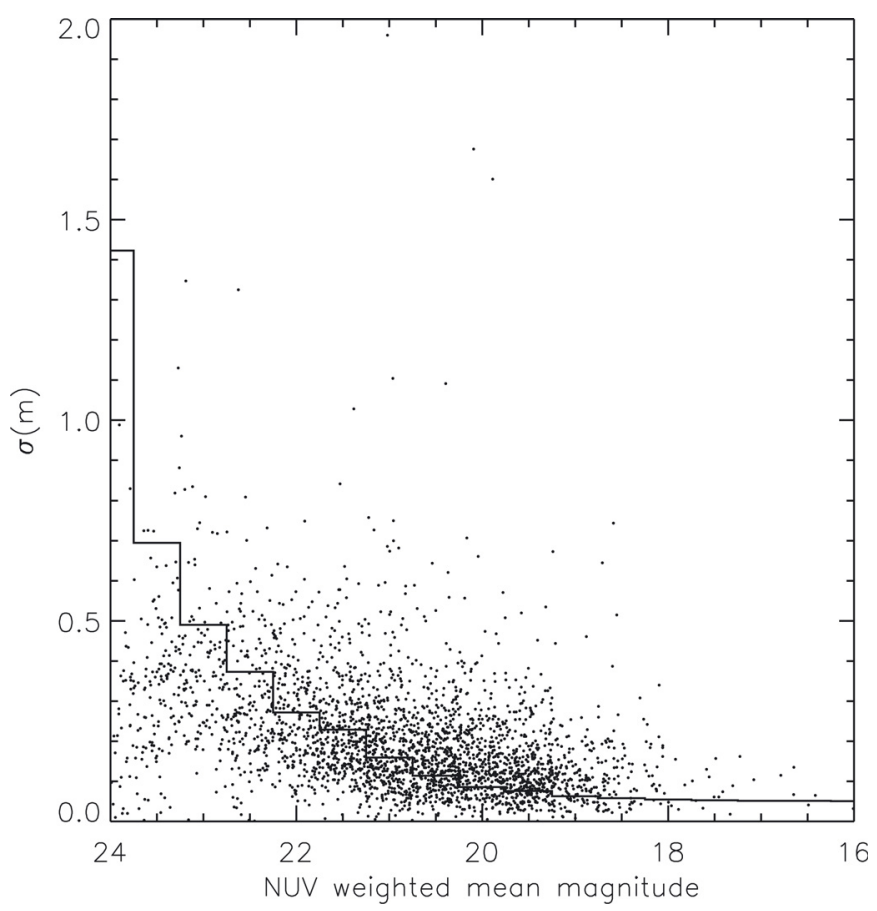

Fig. 4. Plot of the standard deviation $(\sigma)$ from the weighted mean $(\langle m\rangle)$ of a series of observed NUV magnitudes $(m)$ for the sample of quasars with $\geq 5$ observational visits by GALEX. Superposed on this plot (full line) is the theoretical 3- $\sigma$ variance from the measured mean NUV magnitude. Only points above this variance were selected as being statistically variable in the NUV.

of 3521 of the selected quasars. As one might expect, the value of $\sigma(\langle m\rangle)$, which is essentially a measure of the error in the derived mean $A B$ magnitude of a quasar, increases with the faintness of the object under study. All of these 3521 quasars were detected as being optically variable in the SDSS DR5 catalog, and although they also all may be UV variable (though this has never been actually proven in any large study), we have decided to take a conservative approach in selecting only the more UV variable objects for further study in this paper. To this end we have decided to use a threshold cut using a standard deviation of 3- $\sigma$ above the level of $\sigma(\langle m\rangle)$ for a given mean magnitude $(\langle m\rangle)$ to reveal significant UV variability. On application of this threshold cut we find that the smallest detectable magnitude variation is $0.11 \mathrm{mag}$, which was determined for a $16.5 A B$ mag quasar of redshift $z=0.4$.

The 3- $\sigma$ threshold cut is shown superposed in both Figs. 4 and 5 respectively for the NUV and FUV observed magnitudes. Application of this statistical significance selection criterion resulted in the identification of 550 quasars as being NUV variable and 371 quasars as being FUV variable, with the vast majority of the FUV variable objects being common with those identified as variable in the NUV. For simplicity, from now on we term this sub-set of selected variable objects as UVQs (i.e. ultraviolet variable quasar stellar objects). The typical levels of observed UV variability for each of these UVQs, can be best viewed when shown as NUV and FUV light-curves (i.e. the $A B$ magnitude for each visit versus observation date). In Fig. 6 we show the FUV and NUV light-curves for a relatively bright (SDSS $\mathrm{J} 163915.80+412833.6$ ) and a faint (SDSS J221629.33+002340) quasar, that were both repeatedly observed over a 4+ year timeframe with GALEX. It should be noted that faint and variable objects can fall below the GALEX threshold of detection during a series of observations, and hence the derived mean magnitude 


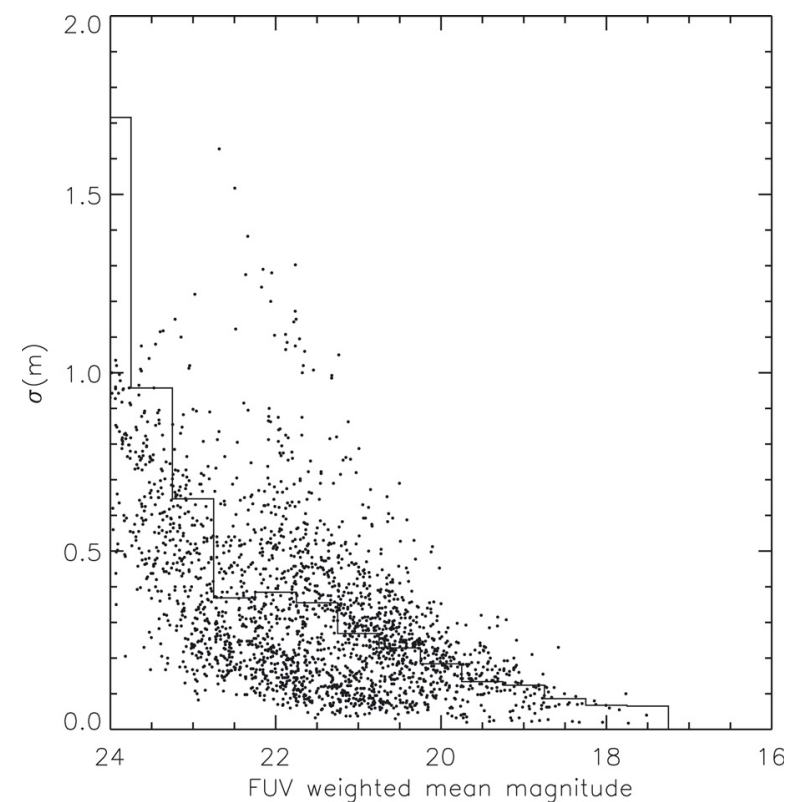

Fig. 5. Plot of the standard deviation $(\sigma)$ from the weighted mean $(\langle m\rangle)$ of a series of observed FUV magnitudes $(m)$ for the sample of quasars with $\geq 5$ observational visits by GALEX. Superposed on this plot (full line) is the theoretical 3- $\sigma$ variance from the measured mean FUV magnitude. Only points above this variance were selected as being statistically variable in the FUV.
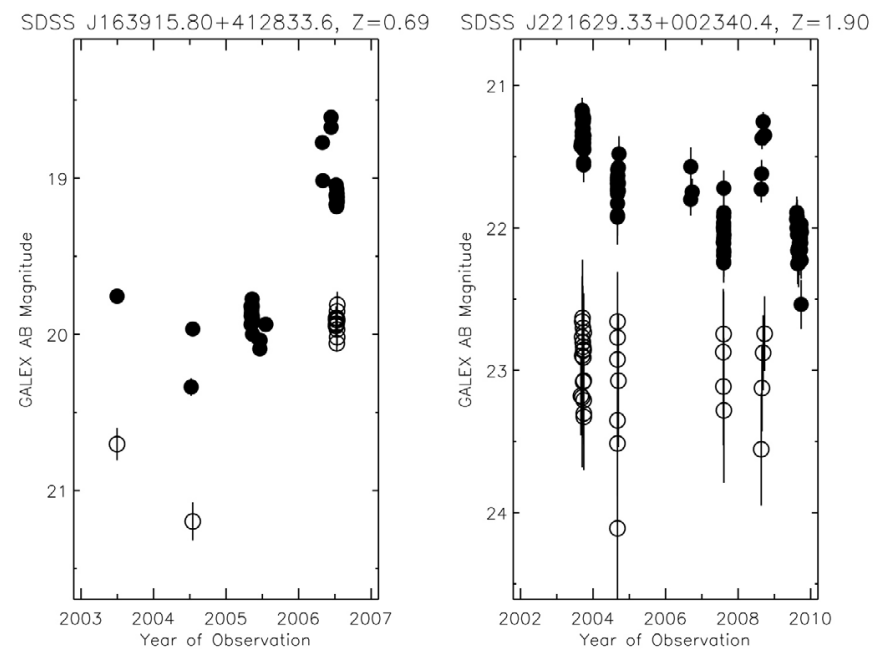

Fig. 6. NUV (filled circles) and FUV (open circles) light curves for the UV-variable quasars (UVQs) SDSS J163915.80+412833.6 (left) and SDSS J221629.33+002340 (right) repeatedly observed over several years with the GALEX satellite. Error bars represent the uncertainty in the measured $A B$ magnitude (see text).

(which is computed from only actual detections within a series of visits) will be of a higher value than had the object been detected during all visits. Thus, the computed change in magnitude, which is a measure of the object's variability, will be underestimated. Finally we note that we did not discover any UVQs that were highly variable in the FUV but not variable in the NUV.

\subsection{The structure function}

The mean variability of UVQs is often described by a Structure Function, which is the correlation between two points in a set of measurements of an object's light curve (Simonetti et al. 1985).
Thus, in its simplest form, it provides a statistical measure of variability as a function of the time-lag between many sets of observations. However we note, as several authors have pointed out, there are limitations in its applicability to derive timescales and amplitudes of quasar variability (Vio et al. 1992). In addition, quasar variability is most probably a complex function of several (possibly interdependent) physical parameters such as luminosity, rest-frame wavelength, redshift, black hole mass and time-lag. Helfand et al. (2001) have pointed out that "the complex interdependence of the observed variability (of quasars) on wavelength, redshift, bolometric luminosity, radio loudness and possibly other parameters makes it difficult to draw definitive conclusions regarding the physics of the central engine or quasar evolution". Thus, "caveat emptor" should be the watchwords for any discussion of quasar variability dependencies, and thus we employ a method by which these dependencies of variability can be plausibly disentangled.

For our present study, in which most of the identified UVQs each have $>5$ measurements, we start by using the standard formulation of the structure function (SF) given by di Clemente et al. (1996):

$$
\mathrm{SF}=\sqrt{\pi / 2 *\langle|\Delta m(\Delta \tau)|\rangle^{2}-\left\langle\sigma_{n}^{2}\right\rangle}
$$

where $\Delta m(\Delta \tau)$ is the difference in UV magnitude between any two observations of a UVQ separated by $\Delta \tau$ in the quasar rest time frame, and $\sigma_{n}^{2}$ is the square of the uncertainty in that difference (being equal to the sum of the two individual observations' errors in quadrature). The final SF for all of our UVQ observations is just the rms of the summation for all possible combinations of magnitude measurements for a given value of time-lag.

In Fig. 7 we show $\log -\log$ plots of the SF (i.e. the measure of UV variability) versus the rest frame time lag (in days) for both the NUV and FUV observations of each of the statistically significant variable UVQs selected from Figs. 4 and 5. Following the work of Vanden Berk et al. (2004) we have plotted the data averages in 10 time-lag bins, chosen with equal intervals in the logarithmic rest-frame time lag. Note that the data as presented in Fig. 7 make no account for any other plausible variability dependencies such as luminosity, redshift or radio loudness. In this form, the variability function is often termed an "ensemble" Structure Function. Error bars were determined by propagating the rms errors derived for the average magnitude difference and measurement uncertainty in quadrature for each observation.

The data shown in Fig. 7 reveal two important facets of the UV variability of quasars. Firstly, the amplitudes of variability in both the NUV and FUV are far larger than those observed at visible wavelengths (which are typically only $\sim 0.03 \mathrm{mag} \mathrm{rms}$ ), and secondly, the levels of FUV variability are greater than those observed in the NUV.

The NUV SF shown in Fig. 7 increases linearly (with a slope of 0.439 ) from a rest frame time-lag of 1 to $\sim 100$ days, with a definite turn-over and subsequent flattening for timelags $\gtrsim 300$ days. The actual best-fit to all of the NUV data is a quadratic in log-log form. A similar correlation of variability with time-lag has also been found in many other studies, and the flattening of the curve at long time-lags has been reported by both Vanden Berk et al.( 2004) and Cristie et al. (1997). However we note that de Vries et al. (2005), in an optical survey of long-term quasar variability up to timescales of $\sim 40$ years, has observed that this apparent flattening of the SF only lasts for a few years before continuing its linear increase with timelag. In essence, they claim no significant turn-over of their SF, such that visible quasar variability increases monotonically with 


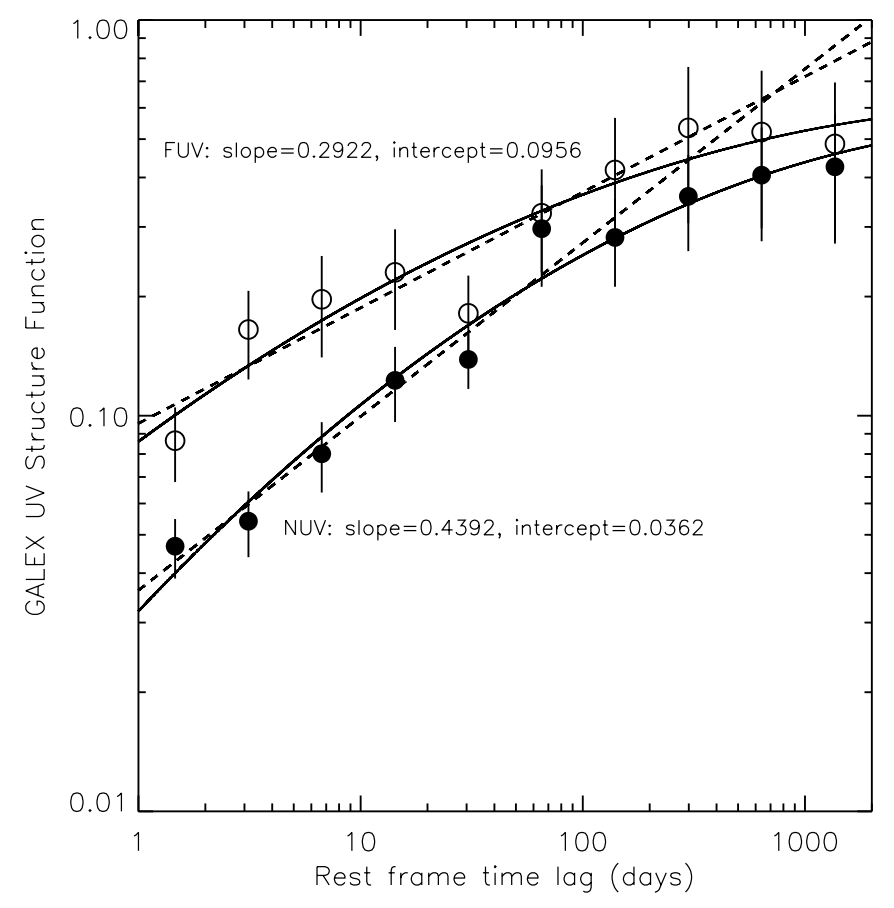

Fig. 7. Log-Log plots of the NUV (filled circles) and FUV (open circles) structure functions with respect to the rest frame time lags for the UV-variable quasars. The data averages are shown in 10 bins of equal logarithmically spaced time lags. Best fit straight lines (dashed) and quadratic fits (full line) are shown for both Structure Functions. Both sets of data reveal a flattening of their structure functions for time lags $>300$ days.

increasingly long time-lags. Although our sampling of UVQs is limited to time-scales of $<5$ years, inspection of the behavior of the NUV SF curve suggests that the rate of increase in UV variability as a function of time decreases when observed over time-lags greater than $\sim 2$ years. The FUV Structure Function, also shown in Fig. 7, follows the same general trend as the NUV curve, except that we have observed a greater level of baseline variability of UVQs in the FUV. However, the change in FUV variability as a function of time-lag is less pronounced than that in the NUV, as indicated by the best-fit line of slope 0.292 . The FUV data, like those recorded in the NUV, also show a flattening in UV variability for time-lags $\gtrsim 300$ days.

\section{Variability correlation plots}

Previous studies of quasar variability in the rest-frame of the optical/UV regime have revealed several apparent physical correlations. For example, trends of variability with wavelength and luminosity (first discovered by di Clemente et al. 1996; and Hook et al. 1994, respectively) have been confirmed by Vanden Berk et al. (2004) who have found that quasars are more variable at shorter rather than longer wavelengths and the amplitude of variability decreases with increasing luminosity (i.e. intrinsically brighter objects vary less). These results, and the SF method used to obtain them, have recently been verified for a sample of quasars observed over a 10 year period by MacLeod et al. (2010). Furthermore, radio-loud quasars appear more variable than their radio-quiet counterparts (Helfand et al. 2001), and the apparent increase in variability for higher redshift quasars is mainly due to the increase of variability with rest-frame frequency and to the increase of rest-frame frequency scanned at high redshift for a given frequency (di Clemente et al. 1996).
The Structure Function derived in the previous section, which described UV variability as a function of rest-frame time-lag, took no account of any of the other possible inter-dependencies such as luminosity or redshift. To reveal such multi interdependenices would require 4-dimensional plots, and thus we need an alternative method to explore other possible correlations with the UV SF. Therefore, we closely follow the work of Vanden Berk et al. (2004) who have described a method that attempts to disentangle these possible physical interdependencies. Since each of the 10 time-lag bins of the UVQs plotted in Fig. 7 contains objects with a wide-range of other (differing) physical parameters, we start by subdividing the targets contained within each time-lag bin into separate (and assumed to be degenerate) physical parameters. This then allows us to obtain a set of (quasiindependent) variability relations with respect to the SF for a particular time-lag, UVQ luminosity, redshift and radio loudness. In the following subsections we present these variability relationships, and then briefly discuss their possible astrophysical meaning in Sect. 4.

\subsection{Time lag - UV variability dependence}

Firstly we explore the dependence of UVQ NUV and FUV variability on the rest frame time-lag, in a form that is (quasi) independent of the parameters of luminosity and radio loudness. We divide all of the UVQs in our UV variability sample, whose redshift $(z)$ values span the range of 0 to 2.5 , into three redshift bins of size $0<z<0.47,0.47<z<1.33$ and $z>1.33$. The physical relevance of these 3 redshift bins with respect to observations through the two GALEX pass-bands will be discussed in Sect. 4. In Figs. 8 and 9 we show log-log plots of the respective NUV and FUV Structure Functions versus rest frame time-lag, with the data averages being plotted separately for each of these three redshift bins at any given rest frame timelag value. Both of these plots, in agreement with the simpler ensemble SFs shown in Fig. 7, show that the amplitudes of both the NUV and FUV variability of UVQs increase as a function of rest frame time-lag, irrespective of the value of quasar redshift for time-lags $<200$ days. For time-lags $>300$ days there is a pronounced rollover in the NUV SF for all redshift values, which is also observed in the lower S/N FUV data. To further demonstrate this roll-over effect we have also included separate linear best-fits for each of the 3 redshift bin data sets in Figs. 8 and 9. These straight-line fits show that rate of increase of the NUV SF up to a time-lag of $\sim 200$ days is greatest for sources of redshift $0.47<z<1.33$, an effect that is also mirrored in the FUV data set of Fig. 9. For time-lags $>300$ days it is clear that the same linear fits are inappropriate for all of the NUV and FUV data, such that a far slower increase in the rate of the NUV and FUV SFs occurs.

Figures 8 and 9 both show that for time-lags $<20$ days there is a definite trend for the higher- $z$ UVQs to be more NUV and FUV variable than their low- $z$ counterparts. This trend has also been seen for visible quasars by de Vries et al. (2005). However, for time-lags $>100$ days this trend mostly disappears such that both the FUV and NUV SF variability amplitudes are similar for all values of UVQ redshift when observed over long time-lags.

\subsection{Luminosity - UV variability dependence}

Unlike redshift, absolute luminosity is an intrinsic physical property of a quasar and thus an investigation of UV variability (i.e. SF) as a function of this parameter should be less affected by 


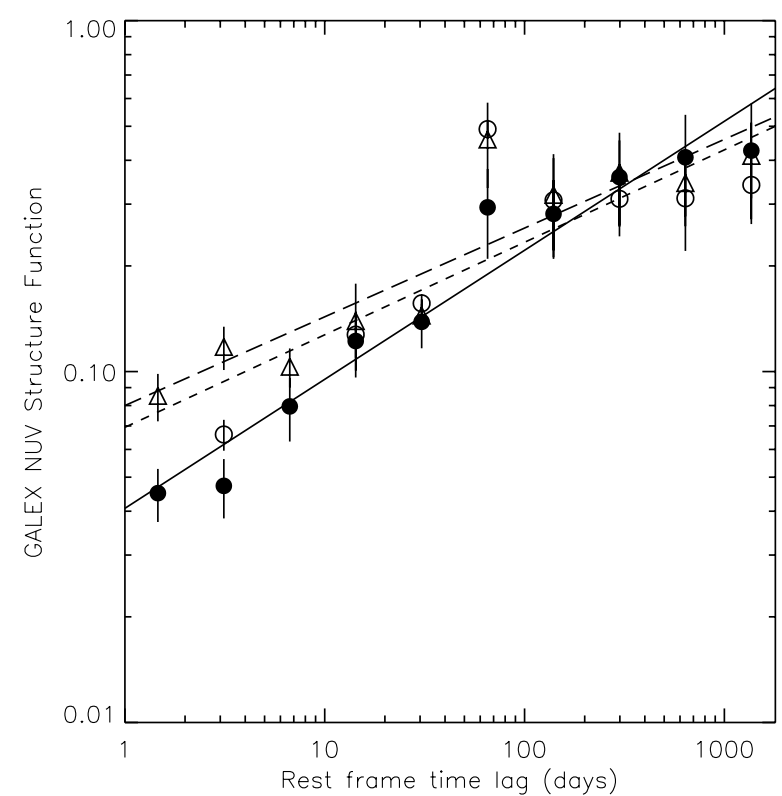

Fig. 8. $\log -\log$ plots of the NUV structure function (separated into 3 redshift bins) with respect to the rest frame time lags for the UV variable quasars. The data are shown for the same 10 bins of time lags as Fig. 7 , but with the data averages plotted for redshift bins of size $0<z<0.47$ (open circles), $0.47<z<1.33$ (filled circles) and $z>1.33$ (open triangles). The vertical lines represent error bars for the binned data points. Linear best-fits are also shown for each of the redshift bin data sets; long dashed line (open circle data), full line (filled circle data) and short dashed line (open triangle data). None of these linear fits match the NUV data for time-lags $>300$ days.

other physical interdependencies. We make use of the values of luminosity given in Schneider et al. (2007) for each of our UV variable sources, and note that these values were calculated using extinction corrected SDSS (absolute) $i$ magnitudes and with the assumption that the quasar spectral energy distribution in the ultraviolet-optical can be represented by a power law.

We start by separating the possible dependency effects of rest frame time-lag by dividing the data into four time-lag bins of 1-10 days, 11-100 days, 101-500 days and >500 days. In Fig. 10 we show $\log -\log$ plots of both the NUV and FUV variability amplitudes (i.e. the SF's) as a function of (averaged) absolute magnitude in bins of 2 mag width for each of the aforementioned time-lag bins. The best-fits to these data show that there is a slight decrease in both the NUV and FUV SFs as a function of increasing luminosity for time-lags >100 days. Similar (visible) luminosity plots have also been constructed by Vanden Berk et al. (2004) for a sample of 25000 quasars in the SDSS survey, in which they similarly found that intrinsically brighter objects vary less than intrinsically fainter ones. However, for shorter time-lags we find the opposite behavior in which the NUV and FUV SFs increase as a function of increasing luminosity (we note the apparent exception of the NUV SF for time-lags $<10$ days, but this may be due to small number statistics with associated larger errors). The NUV and FUV data shown in Fig. 10 also show that variability amplitudes are greater at longer time-lags than at shorter time-lags for all values of UVQ luminosity. This behavior is also revealed in the SFs of Figs. 7-9, which were derived independently of luminosity. This difference in variability for both short and long time-lag values is discussed further in Sect. 4.1 with respect to the work of Kelly et al. (2009).

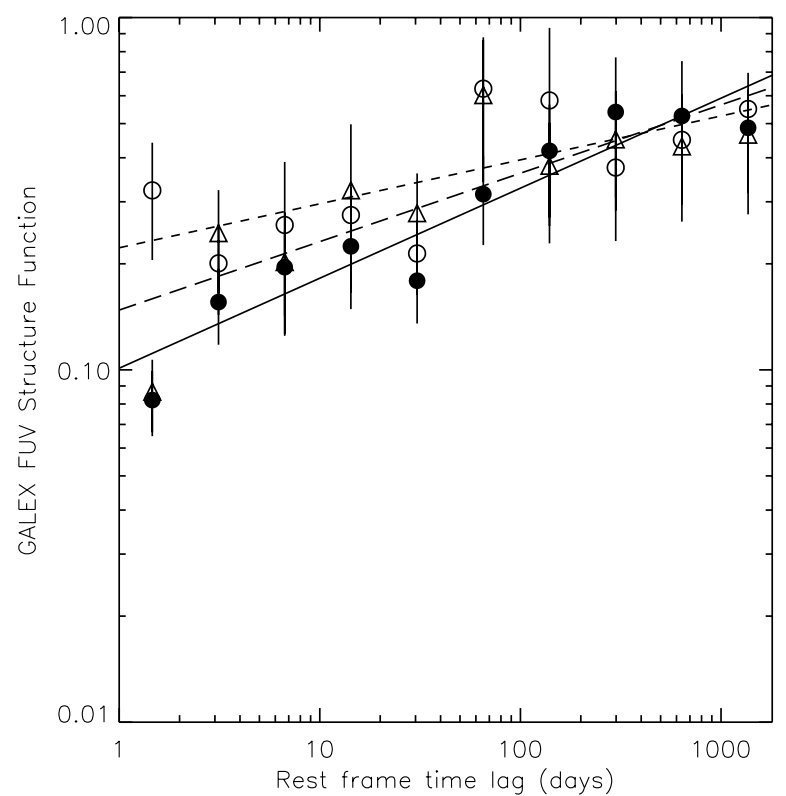

Fig. 9. Log-Log plots of the FUV structure function (in 3 redshift bins) with respect to the rest frame time lags for the variable quasars. The data are shown for the same 10 bins of time lags as Fig. 7, with the data averages plotted for redshift bins of size $0<z<0.47$ (open circles), $0.47<z<1.33$ (filled circles) and $z>1.33$ (open triangles). The vertical lines represent error bars for the binned data points. Linear bestfits are also shown for each of the redshift bin data sets; long dashed line (open circle data), full line (filled circle data) and short dashed line (open triangle data). None of these linear fits match the FUV data for time-lags $>300$ days.

We note that there are no low-luminosity UVQs detected at high redshift in our sample, such that the low luminosity bins in both plots of Fig. 10 are populated mostly by low- $z$ objects. Therefore, since the data in Fig. 10 may still contain some hidden dependency on quasar redshift, in Fig. 11 we re-plot the NUV and FUV variability data but now subdivided into bins of redshift. For convenience of plotting we have selected representative redshift bins of $0.5<z<0.895$ and $z>2.028$ for the NUV data, and bins of $0.5<z<0.895$ and $1.4<z<2.03$ for the FUV data, all plotted for the same two representative time-lag bins as used in Fig. 10. For the NUV data in Fig. 11 the 4 curves of SF versus luminosity do not precisely follow the same trends as shown in Fig. 10, although there is an overall general trend of decreasing SF value with increasing luminosity. This general trend of decreasing variability amplitude is also observed in the FUV data, except for the case of high $z$ and small time-lags where an increase of SF variability with luminosity is observed. Since the general trends seen in both Figs. 10 and 11 are similar, we can deduce that redshift does not seem to have a profound effect on the UV variability- luminosity nature of these objects, and also that the shape of the UV SFs is only weakly dependent on quasar luminosity.

\subsection{Radio loudness - variability dependence}

In Figs. 12 and 13 we plot the SFs for the NUV and FUV variability of our quasar sample against their rest frame time-lag for both radio loud and radio faint UVQs. Based on their peak $20 \mathrm{~cm}$ radio flux density as measured in the VLA FIRST survey and using the absolute SDSS $i$ magnitudes and distances listed in Schneider et al. (2007) to derive a measure of their optical 

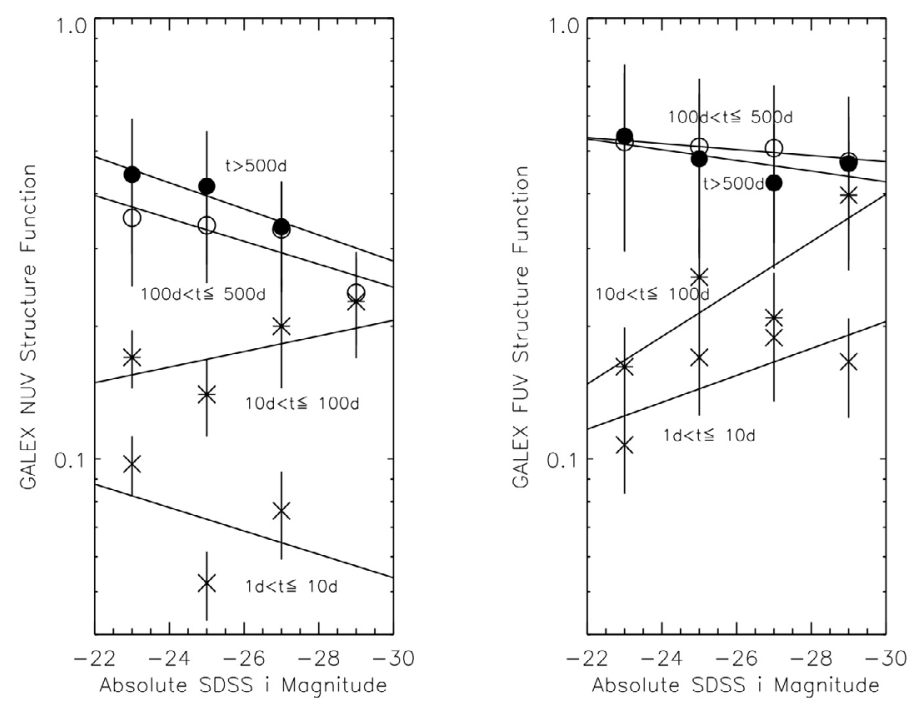

Fig. 10. Plots of the NUV (left) and FUV (right) structure functions as a function of quasar luminosity (absolute $i$ magnitude) for our sample of UV variable objects. The data are plotted in bins of 2 mag width and are shown for four rest frame time-lags $(t)$ of $1 \mathrm{~d}<t \leq 10 \mathrm{~d}$ (crosses), $10 \mathrm{~d}<t \leq 100 \mathrm{~d}$ (asterisks), $100 \mathrm{~d}<t \leq 500 \mathrm{~d}$ (open circles) and $>500 \mathrm{~d}$ (filled circles) for both the NUV and FUV observations. Best-fit lines to the data points are also shown, revealing that NUV and FUV variability amplitudes are greater at longer time-lags than for shorter time-lags for all values of QSO luminosity
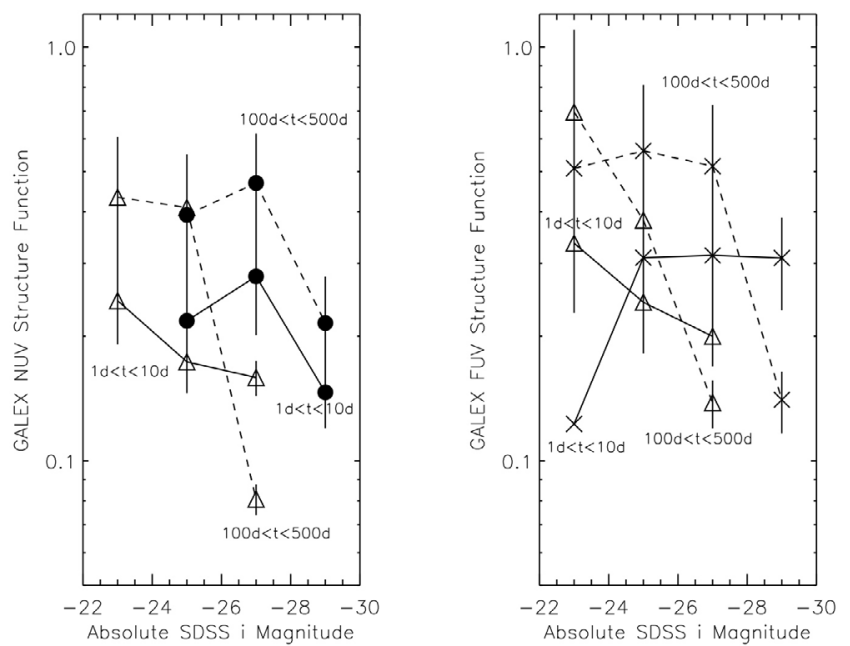

Fig. 11. Log-Log plots of the NUV (left) and FUV (right) structure functions as a function of quasar luminosity (absolute $i$ magnitude) for our sample of UV variable objects for rest frame time-lags of $1 \mathrm{~d}<$ $10 \mathrm{~d}$ and $100 \mathrm{~d}<500 \mathrm{~d}$ for QSOs with redshifts of $0.5<z<0.9$ (open triangles) and $z>2.03$ (filled circles) for the NUV data, and redshifts of $0.5<z<0.9$ (open triangles) and $1.4<z<2.03$ (asterisks). The data points have been connected by a continuous line for the $1 \mathrm{~d}<10 \mathrm{~d}$ data, and by a dashed line for the $100 \mathrm{~d}<500 \mathrm{~d}$ data for ease of viewing.

luminosity, we compute values of the ratio of radio to optical luminosity in the rest frame $\left(R^{*}\right)$. Using Eqs. (1) and (2) of Sramek \& Weedman (1980) to derive $R^{*}$, we use the widely accepted definition of $R^{*}<1$ for radio quiet sources and $R^{*}>1$ for radio loud sources. Since many of our variable quasars do not have listed values for their radio fluxes, our NUV sample is restricted to 484 radio loud and 66 radio quiet sources and our FUV sample contains 327 radio loud and 44 radio quiet sources. Hence,

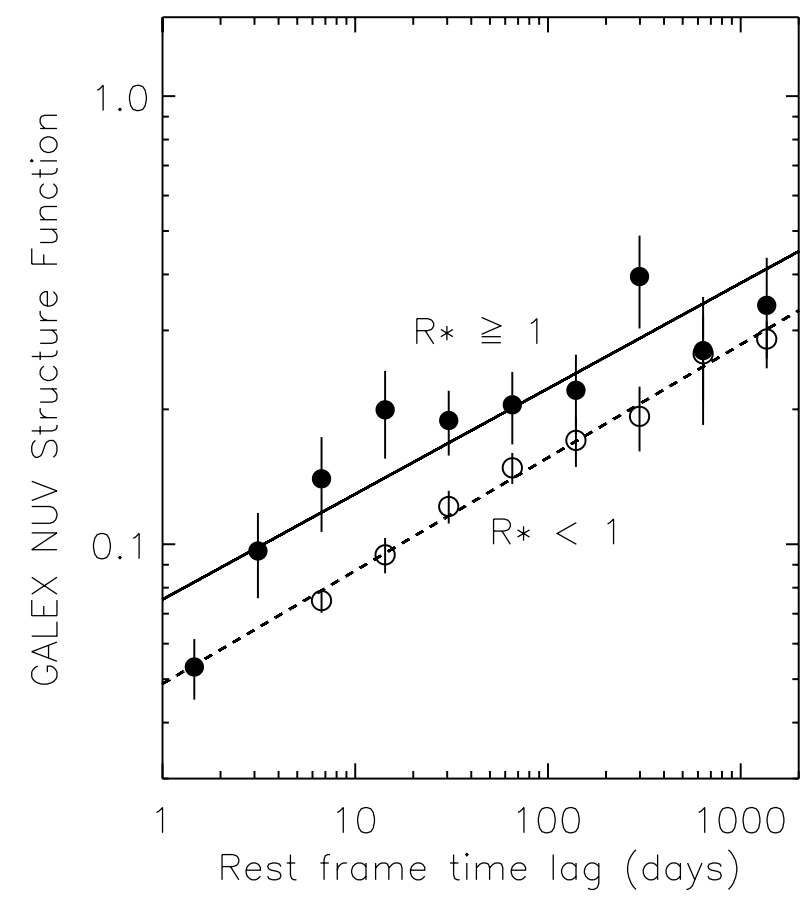

Fig. 12. $\log -\log$ plot of the NUV structure function as a function of rest frame time-lag for radio bright UVQs $\left(R^{*}>1\right.$, filled circles $)$ and radio quiet $\left(R^{*}<1\right.$, open circles) UVQs, as detected by the VLA $20 \mathrm{~cm}$ FIRST survey. Best fit straight lines are shown for each of the two data sets. The radio bright NUV data are best fit with a line of slope 0.25 (intercept 0.05) and the radio faint sources with a similar slope of 0.24 (intercept 0.08).

the reader is reminded that we are dealing with small number statistics for the radio quiet source data in both Figs. 12 and 13.

Firstly, inspection of both figures clearly shows that the overall level of SF variability is greater in the FUV than the NUV for both radio loud and radio quiet UVQs. This is merely a restatement of the behavior of the entire UVQ sample, as shown in Fig. 7. However Fig. 12 also indicates that radio loud sources are $\sim$ a factor 2 more NUV variable as a function of time-lag than radio quiet sources, and the plot also shows a larger scatter in the shape of the NUV SF for radio loud than for radio quiet UVQs. However, there is only a marginal difference between the best-fit slopes to these NUV SF data for both types of radio source. Therefore, our data would appear to support the notion that NUV variability is not strongly linked to the radio properties of UVQs, and as such, the physical mechanism responsible for the NUV variability of radio detectable UVQs operates irrespective of their radio flux.

The FUV data shown in Fig. 13 have far fewer measurements and larger errors than the NUV data, but again the radio loud UVQs appear to be more variable as a function of time-lag (especially for periods $>100$ days) than the radio quiet sources. The best-fit slope of 0.16 to the FUV radio loud data is significantly different from that of the best-fit slope of 0.059 for the radio quiet data. This FUV variability difference is most pronounced for time-lags $>100$ days. However, we note that this conclusion is based on only a small number of UVQs. We note that several authors have claimed that radio-loud quasars are (marginally) more variable than their radio-quiet counterparts (Eggers et al. 2000; Helfand et al. 2001), whereas Bauer et al. (2009) have found no evidence for any change in the optical variability amplitude of quasars with increasing radio loudness (luminosity). Several studies have found that optical microvariability, operating over 


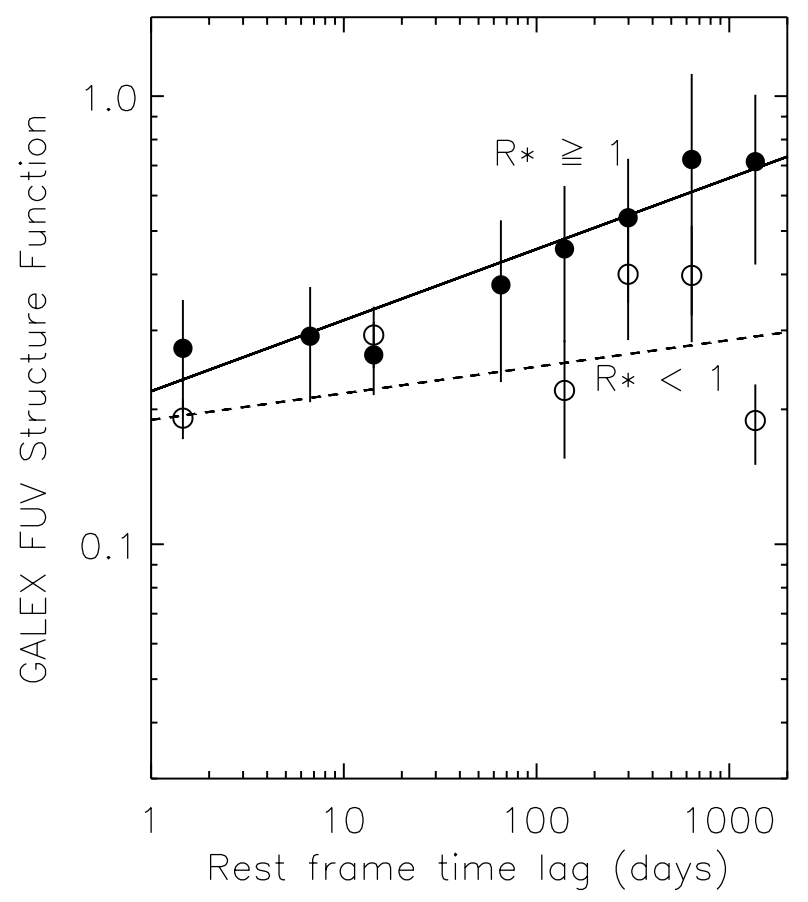

Fig. 13. $\log -\log$ plot of the FUV structure function as a function of rest frame time-lag for radio bright UVQs $\left(R^{*}>1\right.$, filled circles) and radio quiet UVQs $\left(R^{*}<1\right.$, open circles) as detected by the VLA $20 \mathrm{~cm}$ FIRST survey. Best fit straight lines are shown for each of the two data sets. The radio bright FUV data are best fit with a line of slope 0.16 (intercept 0.22 ) and the radio faint sources with a slope of 0.059 (intercept 0.19$)$.

time-scales of minutes to hours, occurs in quasars regardless of their radio properties (Ramirez et al. 2009). Our present NUV (and FUV) observations support the notion in which radio loud UVQs are only marginally more UV variable than radio quiet sources, irrespective of the time-frame of observation.

\section{Summary discussion}

Before briefly discussing any physical interpretation of the correlations shown in Figs. $7-13$ of Sect. 3, we first provide a short summary of the underlying UV properties of the UVQ sample. We also remind the reader that due to our conservative approach in the selection of UV variable quasars, these findings may only apply to the more UV variable of this class of object. In Fig. 14 we show a typical quasar rest-frame spectrum (adapted from Vanden Berk et al. 2001), which shows the prominent $\operatorname{Ly} \alpha$, CIV, CIII and MgII emission lines superposed on a significant underlying UV continuum level, which for wavelengths blueward of $\operatorname{Ly} \alpha$ is heavily extinguished by the Ly $\alpha$ forest of absorption lines. The entire UV energy distribution is often referred to as the "big blue bump", and is thought to be dominated by the $10^{4-5} \mathrm{~K}$ thermal emission from a central black hole accretion disk. Based on the sensitivity and observational strategy of the GALEX satellite, the great majority of quasars that can be detected possess redshifts in the range $0 \leq z \leq 2.5$ (Bianchi et al. 2007). For quasars with redshifts in the range from $z=0.11$ to 0.47 the strong $\operatorname{Ly} \alpha$ emission line is present within the GALEX FUV passband, and for redshifts of $z=0.46$ to 1.33 the Ly $\alpha$ line is present within the NUV passband. In addition, the Ly $\alpha$ forest of absorption lines (which considerably reduces the UV flux in a quasar spectrum) affects the entire GALEX passbands for redshifts of $z>1.33$. Therefore,

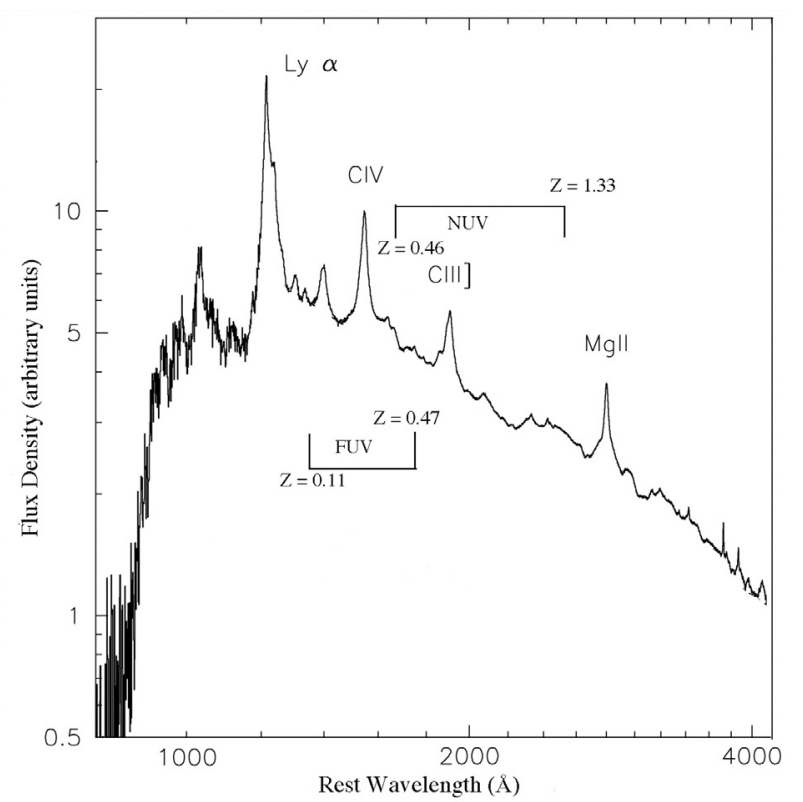

Fig. 14. UV-visible spectrum of a zero redshift quasar showing the most prominent emission lines. Also shown are the GALEX FUV and NUV passbands together with the redshift $(z)$ values at which Ly- $\alpha$ emission enters and leaves each of the passbands (adapted from Vanden Berk et al. 2001)

any observed UV variability within either of the GALEX FUV or NUV passbands will be highly dependent on which of the emission/absorption/continuum features present in the quasar spectral energy distribution have been redshifted into, or out of, those two passbands and also which of these features are deemed to be most responsible for the physical origin of the observed UV variability. Therefore, our selection of 3 redshift bins $(0.11<z<0.47,0.47<z<1.33$ and $z>1.33)$ for the plotting of data in Figs. 8 to 9 represent the appearance and disappearance of the strong Ly- $\alpha$ line in the FUV and NUV bands. Additionally, because of time dilation effects, high redshift objects are potentially observed for less time than low redshift ones. This will possibly affect any study of the time-lag on UVQ variability, which is why we have binned our time-lag data in the rest frame.

Presently, explanations of the primary cause of quasar variability can be broadly split into 3 classes: (i) gravitational microlensing; (ii) discrete-event (Poissonian) processes and (iii) accretion disk instability models. Vanden Berk et al. (2004) have discussed these various processes (see the many references therein) and their visible data favor an accretion disk instability (or jet) cause of the observed optical variability. Since many of our present results (shown in Figs. 7-13) generally agree with the visible observations of quasar variability by Vanden Berk et al. (2004), we shall only address whether a model involving accretion disk instabilities may explain our UV variability data. However, the reader should note that no theoretical model, irrespective of its underlying physical mechanism, currently exists that can be directly compared with their (or our own UV) observations.

Long-term $(7.5 \mathrm{yr})$ changes in the UV continuum level of order $25-125 \%$ have been reported for low luminosity quasars (Kaspi et al. 2000), which is about twice the continuum variability observed over a similar period for high luminosity (high-z) objects (Kaspi et al. 2007). Interestingly for this latter sample of (11) quasars, none showed Ly- $\alpha$ emission variations to a 
level of $<7 \%$ over the 6 -year period of observations. A similar case of non-variability of the Ly- $\alpha$ line has also been reported for observations of 3C 273 recorded over a $15 \mathrm{yr}$ timeframe by Ulrich et al. (1993). Several authors have claimed that quasar continuum variations are dominated by processes that are intrinsic to the accretion disk (Peterson et al. 2004), and Trevese, Kron \& Bunone (2001) have suggested that the observed spectral changes are consistent with temperature variations of a blackbody of $\sim 2.5 \times 10^{4} \mathrm{~K}$ that emits in the UV. More recently Pereyra et al. (2006) have also found that most of the optical-UV variability observed in quasars is due to changes in the UV continuum level which can be directly attributed to processes involving changes in the disk mass accretion rates. Repeatedly visible spectroscopic observations of 2500 quasars over $>50$ day time-frame by Wilhite et al. (2005) have shown that the strongest quasar emission lines vary by only $30 \%$ as much as the underlying continuum level. All these results are therefore consistent with the increasingly widely-held belief that the majority of a quasar's UV-optical spectrum arises directly from the accretion disk and that changes in the level of the underlying UV continua of quasars are probably the main driver of the observed variability in these objects.

\subsection{Ensemble structure function dependence}

The ensemble Structure Functions shown in Fig. 7 clearly reveal that both the FUV and NUV variability of UVQs are well correlated with rest frame time-lags of $\$ 300$ days, such that the UV variability amplitude increases almost linearly with increasing time-lag. However, for longer time-lags there is a rollover in both SF curves, which is also observed at visible wavelengths. It is therefore initially tempting to attribute this rollover to a change in the underlying physical process that cause the observed UV variability. However, a rollover in the SF at large time-lags has recently been fully explained by the "damped random walk" model of quasar variability presented by Kelly et al. (2009) and MacLeod et al. (2010). These authors model quasar light curves as a continuous time stochastic process, which results in the estimation of a characteristic timescale and amplitude for visible flux variations. In the visible, these flux variations are thought to result from thermal fluctuations that are driven by an underlying stochastic process such as a turbulent magnetic field associated with the black hole accretion disk. The shape of their power spectrum (i.e. SF) is predicted to be $1 / f^{2}$, which then flattens to white noise at timescales long compared to the relaxation time. This relaxation time is interpreted (for quasar flux variations) as the time required for the time series of fluctuations to become roughly uncorrelated. From our present NUV and FUV data this relaxation time is $\sim 400$ days.

The reader should be reminded, however, that our GALEX observations are biased towards the detection of brighter and lower-redshift objects which could potentially be making a larger contribution to the ensemble SF at long time lags compared with the higher redshift (and fainter) UVQs. Hence, although the ensemble SFs shown in Fig. 7 are important indicators of quasar variability, only by discussing the potential dependencies shown Figs. 8-13 can we better interpret these data.

\subsection{Time lag dependence}

The NUV and FUV SFs shown in Figs. 8 and 9 as a function of time-lag follow the same general trend as their ensemble SF counterparts shown in Fig. 7, in that the amplitude of
UV variability increases (linearly) as a function of rest frame time-lag up to $\sim 200$ days and thereafter there is a pronounced rollover in the NUV and FUV SFs for all redshift values. This overall trend is similar, irrespective of the value of UVQ redshift. For short time-lags $<20$ days there is a definite trend for higher- $z$ UVQs to be more NUV and FUV variable than their low- $z$ counterparts, but this trend all but disappears for time-lags in excess of 100 days. If we assume that the observed variability is mainly due to changes in UV line emission, then we might expect the NUV band to exhibit greater variability than that recorded in the FUV band for objects with $z=0.46$ to 1.33 (i.e. the range in which the strong Ly- $\alpha$ line enters the NUV band pass). However, our observations do not strongly support this notion since the SF variability levels of the FUV data for these objects are similar to those of their NUV variability. Thus, it seems likely that the observed FUV variability for the UVQs in this redshift range may be better explained through (stochastic) changes in the underlying UV continuum level.

\subsection{Luminosity dependence}

Although it is generally agreed that the intrinsic luminosity of a quasar may be linked to matter accreting from a disk onto a black hole, the physical mechanism(s) responsible for the observed changes in variability are still widely debated. As pointed out in the Introduction, possible variability mechanisms from extrinsic gravitational sight-line microlensing to intrinsic accretion disk instabilities have been forwarded to explain the observations. Using IUE satellite observations, Paltani \& Courvoisier (1997) found that quasar variability and luminosity (absolute magnitude) were related by a power law slope of -0.08 , such that relative variability decreases as the quasar luminosity increases. This anticorrelation between quasar variability and luminosity has also been confirmed at optical wavelengths using large homogeneous data sets (Bauer et al. 2009; Vanden Berk et al. 2004). However, we note that Ai et al. (2011) claim that the inverse dependence of variability on luminosity is not significant for a sample of SDSS selected AGN (mostly quasars). They claim that their analysis was capable of recovering AGN with a small variability in the low luminosity regime which were largely missed in most of the previous studies. Furthermore, previous relationships between quasar luminosity and other parameters such as black hole mass and spectral line-widths have mostly been derived from single-epoch estimates and using (mostly untested) extrapolations from low to high redshift objects (Vestergaard \& Peterson 2006).

Our present findings shown in Fig. 10 indicate that there is a general decrease in both NUV and FUV variability as a function of increasing absolute magnitude for time-lags $>100 \mathrm{~d}$. The opposite effect is observed for time-lags $<100 \mathrm{~d}$ (apart from the weak exception in the NUV for time-lags $<10 \mathrm{~d}$ ). Figure 11 shows that this general decrease in UV variability as a function of luminosity is not strongly coupled to the UVQ redshift, in conflict with other (visible) studies that have claimed a redshiftvariability relationship (Trevese \& Vagnetti 2002). Both of the NUV and FUV SFs shown in Fig. 10 reveal a similar behavior to those shown in Figs. 7-9 (which were derived independently of luminosity), in that variability amplitudes are greater at longer time-lags than for shorter time-lags for all values of QSO luminosity. Therefore, it would seem from our present data that the intrinsic luminosity of a UVQ may only be weakly connected to the origin of the physical mechanism causing the UV variability, and that this mechanism operates over most time-lags and is not strongly dependent of the UVQ redshift. As discussed in 
Sect. 4.1, although the actual underlying physical causes have yet to be proven, the application of a "damped random walk" model of stochastic thermal fluctuations from an accretion disk can statistically explain the general shape of the ensemble variability of quasars, and in particular the shape of their SFs as a function of time-lag (Kelly et al. 2009; MacLeod et al. 2010). Therefore, in order to explain the majority of our UV variability data we do not have to invoke various physical mechanisms that operate over different time-lags that depend on various physical parameters, but instead the gross behavior of the UV variability of quasars can essentially be described by a $1 / f^{2}$ dependence that flattens to a near constant at low frequencies (i.e. long time-lags). This seems to be true apart from the effect noted in Sect. 4.2 (and shown in Figs. 8 and 9) in which higher- $z$ UVQs appear to be more variable than their low- $z$ counterparts for time-lags $<20$ days. Whatever physical (or observational) effect is responsible for this behavior essentially disappears from our data for time-lags $>100$ days.

\subsection{Radio loudness - UV variability}

Although the data presented in both Figs. 12 and 13 have been constructed with relatively few UVQs, we note the near similarity between the shape of the NUV SFs for both radio loud and radio quiet objects. In addition, we note that both figures also show that radio loud UVQs are marginally more variable than radio-quiet sources (a factor 2 in the NUV and a factor 1-3 in the FUV, depending on time-lag), in agreement with the work of both Eggers et al. (2000) and Helfand et al. (2001). Similarly, several other visible variability studies (Vanden Berk et al. 2004; Ramirez et al. 2009) have revealed a correlation between radio loudness and UV/optical variability amplitude that was suggestive but not conclusive. The source of quasar radio emission is thought to originate either in an accretion disk around the central black hole or in shock waves in a relativistic jet ejected from a region around the central black hole. Since the processes associated with a powerful nuclear radio source probably influence UV variability over time-lags $\lesssim 100$ days, we might expect the greatest difference in variability between radio loud and radio quiet objects to appear in this time-regime. Both of our FUV and NUV data do not support such a scenario, and to the contrary the (sparsely sampled) FUV SF suggests that the greatest variability difference occurs for time lags $>100$ days for radio sources. Thus, although our data do reveal marginal differences between the behavior of radio loud and radio quiet sources, we believe that the radio properties of UVQs appear only play a minor role in the gross levels of observed UV variability.

\section{Conclusion}

Using archival observations recorded at NUV and FUV wavelengths over a 5+ year timeframe with the NASA GAlaxy Evolution eXplorer (GALEX) satellite, we have performed an ultraviolet variability analysis of quasars that have optical counterparts in the Sloan Digital Sky Survey (SDSS) DR5 spectroscopic catalog of Schneider et al. (2007). The application of a set of (conservative) selection criteria has resulted in the identification of 550 quasars in the NUV and 371 in the FUV as being statistically significantly variable using data recorded in 5 or more GALEX observations spread over time periods $>3$ months. Typically we have observed a wide variety of UV variability levels ranging from 0.11 to $3.0 A B$ magnitudes arising in quasars with redshifts of $0<z<2.5$.
In order to explore possible correlations within these variability data we have used a Structure Function analysis that closely follows the work of Vanden Berk et al. 2004, who performed a similar analysis on visible observations of SDSS quasars. By subdividing the variable quasars into time-lag and/or redshift bins we have been able to obtain a set of (quasiindependent) variability relations with respect to the Structure Function (SF) for values of rest frame time-lag, luminosity and radio loudness. Since our selection criteria for UV variability was quite conservative, our analysis of the SFs may only be applicable to the more UV variable QSOs.

The SF-time-lag plots clearly reveal that the amplitudes of variability in both the NUV and FUV are far larger than those observed in quasars at visible wavelengths. Additionally, the levels of FUV variability are greater than those observed in the NUV at any given value of time-lag. We also find that the amplitudes of both the NUV and FUV variability of UVQs increase as a function of rest frame time-lag, irrespective of the value of quasar redshift, for time-lags $<200$ days. For time-lags $>300$ days there is a pronounced rollover in the NUV SF for all redshift values, which is also observed (with a lower significance) in the FUV variability data. These data also show that for time-lags $<20$ days there is an apparent trend for higher- $z$ UVQs to be more NUV and FUV variable than their low- $z$ counterparts. However, none of of other plots of SF dependence show any strong dependence on redshift, and this effect may be due to some other physical dependency. Overall, these results are best interpreted under the assumption that the UV variability is linked to variations in the level of continuum, and not line, emission.

Our data also indicate that for time-lags $>100$ days the more luminous UVQs tend to be less NUV and FUV variable, whereas the opposite effect is mostly found for shorter time-lags (with the weak exception in the NUV for lags of $<10$ days). This difference in the level of SF variability between long and short timelags is also seen in the ensemble SF-time lag plots, which were derived independently of luminosity. We also find that UVQ redshift does not seem to have a profound effect on any of the variability-luminosity relations (alhtough we note the previously mentioned trend for higher- $z$ UVQs to be more NUV and FUV variable than their low- $z$ counterparts as a function of short timelags). The apparent weak effect of redshift on the UV variabilityluminosity is perhaps not surprising since luminosity is an intrinsic physical property whereas redshift is not. These data can possibly be interpreted through the application of a "damped random walk" model of stochastic thermal fluctuations from an accretion disk which can statistically explain the general shape of our VUV SFs ((Kelly et al. 2009; MacLeod et al. 2010).

Although our data set is small, it seems that radio loud sources are marginally more NUV variable as a function of time-lag than radio quiet UVQs. Therefore it would seem that whatever the main mechanism is responsible for the observed NUV variability in quasars, it seems to operate irrespective of their radio flux. Such behavior can also be explained by the damped random walk model. Our data also hints that for timelags $>100$ days radio loud sources may be more FUV variable than radio quiet sources, although this conclusion is based on small number statistics.

In summation, our analysis favors a quasar model in which UV variability is mainly due to (stochastic) changes in the underlying continuum level, rather than models that favor gravitational microlensing or discrete-event processes. Finally, we note that as yet, no detailed quasar model currently exists that can be directly compared with our observations of NUV and FUV variability. 
Acknowledgements. We particularly acknowledge the dedicated work of the GALEX mission operations support staff at JPL/Caltech in Pasadena. Financial support for this research was provided by the NASA GALEX Guest Investigator program and NASA grant NAS5-98034 to UC Berkeley. We are indebted to Dr. Suvi Gezari who made many suggestions that have greatly improved this paper.

\section{References}

Ai, Y., Yuan, W., Zhou, H., et al. 2011, ApJ, 727, 31 Bachev, R. S. 2009, A\&A, 493, 907

Bauer, A., Baltay, C., Coppi, P., et al. 2009, ApJ, 696, 1241

Bertin, E., \& Arnouts, S. 1996, A\&AS, 117, 393

Bianchi, L., Rodriguez-Merino, L., Viton, M., et al. 2007, ApJS, 173, 659

Bianchi, L., Hutchings, J., Efremova, B., et al. 2009, AJ, 137, 3761

Courvoisier, T., \& Ulrich, M. 1985, Nature, 316, 524

Cristiani, S., Trentini, S., La Franca, F., \& Andreani, P. 1997, A\&A, 321, 123

de Vries, W., Becker, R., White, R., \& Loomis, C. 2005, AJ, 129, 615

di Clemente, A., Giallongo, E., Natali, G., et al. 1996, ApJ, 463, 466

Eggers, D., Sahffer, D., \& Weistrop, D. 2000, AJ, 119, 460

Gezari, S., Basa, S., Martin, D. C., et al. 2008, ApJ, 676, 944

Giveon, U., Maoz, D., Kaspi, S., et al. 1999, MNRAS, 306, 637

Helfand, D., Stone, R., Willman, B., et al. 2001, AJ, 121, 1872

Hook, I., McMahon, R., Boyle, B., \& Irwin, M. 1994, MNRAS, 268, 305

Kaspi, S., Smith, P., Netzer, H., et al. 2000, ApJ, 533, 631

Kaspi, S., Brandt, W., Maoz, D., et al. 2007, ApJ, 659, 997

Kelly, B., Bechtold, J., \& Siemiginowska, A. 2009, ApJ, 698, 895

Kozlowski, S., Kochanek, C., Udalski, A., et al. 2010, ApJ, 708, 927

MacLeod, C., Ivezic, Z., Kochanek, C., et al. 2010, ApJ, 721, 1014
Martin, D. C., Fanson, J., Schiminovich, D., et al. 2005, ApJ, 619, L1 Miller, H. R., Carini, M., \& Goodrich, B. 1989, Nature, 337, 627 Morrissey, P., Conrow, T., Barlow, T., et al. 2007, ApJS, 173, 682 Paltani, S., \& Courvoisier, T. 1994, A\&A, 291, 74

Paltani, S., \& Courvoisier, T. 1997, A\&A, 323, 717

Pereyra, N., Vanden Berk, D., Turnshek, D., et al. 2006, ApJ, 642, 87

Peterson, B. 1993, PASP, 105, 247

Peterson, B. M., Ferrarese, L., Gilbert, K., et al. 2004, ApJ, 613, 682

Ramirez, A., de Diego, J., Dultzin, D., \& Gonzalez-Perez, J.-N. 2009, AJ, 138, 991

Ramirez, A., Dultzin, D., \& de Diego, J. 2010, ApJ, 714, 605

Schneider, D., Hall, P., Richards, G., et al. 2007, AJ, 134, 102

Sesar, B., Ivezic, Z., Lupton, R., et al. 2007, AJ, 134, 2236

Simonetti, J., Cordes, J., \& Heeschen, D. 1985, ApJ, 296, 46

Sramek, R., \& Weedman, D. 1980, ApJ, 238, 435

Stalin, C., Gupta, A., Gopal-Krishna, Wiita, P., \& Sagar, R. 2005, MNRAS, 356, 607

Trammell, G., Vanden Berk, D., Schneider, D., et al. 2007, AJ, 133, 1780

Trevese, S., \& Vagnetti, F. 2002, ApJ, 564, 624

Trevese, D., Kron, R., \& Bunone, A. 2001, ApJ, 551, 103

Ulrich, M.-H., Courvoisier, T., \& Wamsteker, W. 1993, ApJ, 411, 125

Ulrich, M.-H., Maraschi, L., \& anhd Urry, C. M. 1997, ARA\&A, 35, 445

Vanden Berk, D., Richards, G., Bauer, A., et al. 2001, AJ, 122, 549

Vanden Berk, D., Wilhite, B., Richard, G., et al. 2004, ApJ, 601, 692

Vestergaard, M., \& Peterson, B. 2006, ApJ, 641, 689

Vio, C., Christiani, S., Lessi, O., \& Provenzale, A. 1992, ApJ, 391, 518

Wheatley, J., Welsh, B. Y., \& Browne, S. 2008, AJ, 136, 259

Wilhite, B., Vanden Berk, D., Kron, R., et al. 2005, ApJ, 633, 638

Wilhite, B., Brunner, R., Grier, C., et al. 2008, MNRAS, 383, 1232

Wold, M., Brotherton, M., \& Shang, Z. 2007, MNRAS, 375, 989 